\title{
Die Dialektik des Erfolgs
}

\author{
Harald Walach \\ Institut für transkulturelle Gesundheitswissenschaften, Europa-Universität Viadrina, Frankfurt/O., Deutschland
}

Komplementärmedizin ist beliebt, nachgefragt und breitet sich aus. Alle Umfragen belegen dies. Im Rahmen des ersten EU-geförderten Projekts, «CAMbrella», zu dem in Kürze ein Supplement der Forschende Komplementärmedizin erscheinen wird, hat eine systematische Übersicht über die Inanspruchnahme von Komplementärmedizin in Europa gezeigt, dass eine große Anzahl von Bürgern in Europa Komplementärmedizin verwendet und ihr gewogen ist; die Tendenz ist mit bis zu $86 \%$ steigend [1, 2]. In geradezu grotesker Ignoranz hierzu verharrt der Mainstream in der Meinung, Komplementärmedizin sei keine wissenschaftlich abgesicherte Form der Medizin, werden Forschungsergebnisse marginalisiert und Forschungskapazitäten auf Themen des Mainstreams beschränkt. Das Meiste, was es in Europa an Forschung zum Thema Komplementärmedizin gibt, stammt, wenn es nicht gerade von der pharmazeutischen Industrie gefördert ist, aus der Förderung durch gemeinnützige Stiftungen oder Versicherungen und nur sehr wenig aus Mitteln der öffentlichen Hand. Dennoch hat sich durch die Forschungen eines wachsenden Kreises von Autoren, die sich komplementärmedizinischer Themen angenommen haben, mittlerweile ein doch erstaunliches Korpus an Erkenntnissen angesammelt. Puristen beharren noch immer darauf, dass in vielen Fällen nicht geklärt sei, ob die Effektivität komplementärmedizinischer Maßnahmen nicht einfach nur auf Placeboeffekte zurückzuführen sei. Die Insinuation hierbei lautet natürlich: dann sind die Effekte ja wertlos. Pragmatiker haben aber oftmals längst erkannt: Ob die Effekte Placeboeffekte oder spezifische Effekte sind, ist einerlei. Wichtig ist, ob die Interventionen den Patienten nachdrücklich und nachhaltig helfen und ob die Hilfe vergleichsweise kostengünstig und nebenwirkungsfrei ist. Ich will ein Beispiel anführen: Im Mai 2009 veröffentlichte der englische Regulator, das National Institute of Clinical Excellence (NICE), der die Vorgaben trifft für das, was im englischen Gesundheitssystem aus öffentlicher Kasse bezahlt werden darf, eine Direktive zur Behandlung von chronischen
Rückenschmerzen. Diese, so NICE, dürften in Zukunft nicht mehr mit Schmerzmitteln behandelt werden, sondern, in dieser Reihenfolge, durch Bewegung, Chiropraktik und Manipulation sowie Akupunktur [3]. Das war mutig, bahnbrechend und sehr konsequent. Die Gutachter von NICE haben gemerkt: Konventionelle Schmerzmittel sind im chronischen Schmerzfall unbrauchbar. Das belegen seit langer Zeit viele Übersichtsarbeiten [4-6]. Sie verursachen darüber hinaus viele Nebenwirkungen, und diese Kategorie von Medikamenten ist auch diejenige, die insgesamt für prozentual die meisten tödlichen Nebenwirkungen von Medikamenten verantwortlich ist. Die Gutachter von NICE haben auch gemerkt: Im Vergleich mit konventionellen Behandlungen sind die Effektgrößen der komplementärmedizinischen Interventionen groß. NICE ist eine pragmatisch vorgehende Health-Technology-Assessment-Behörde. Sie fragt nach dem therapeutischen Nutzen und Mehrwert, sauber wissenschaftlich belegt. Ob dieser Mehrwert dabei auf nachvollziehbaren mechanistischen Bahnen erzeugt wird - die wissenschaftliche Perspektive - oder auf unbekannten Wegen, vielleicht sogar über rein psychologische Placeboeffekte, ist dem Regulator egal. Sir Michael Rawlins, der Direktor von NICE, hat einmal in einem öffentlichen Vortrag vor der King's Foundation in London und auch in seiner Harveian Oration, einem Festvortrag vor dem Royal College of Physicians, gesagt, ihn als Pragmatiker interessiere nicht, ob ein Effekt auf verstandenen pharmakologischen Wegen oder auf unverstandenen zustande käme. Hauptsache, es ist ein wägbarer Effekt, der dem einer konventionellen Behandlung in nichts nachsteht oder besser ist - Placebo hin oder her. Im Klartext: Der Effekt muss wissenschaftlich, also durch randomisierte, kontrollierte Studien, belegt sein; er muss sich gegenüber der gängigen Standardtherapie als ebenbürtig und billiger oder nebenwirkungsärmer zeigen oder aber als besser [7]. Dies ist im Falle der Behandlung chronischer Rückenschmerzen bei den besagten komplementärmedizinischen Verfahren wissenschaft-

\section{KARGER}

Fax +497614520714

Information@Karger.de

www.karger.com (c) 2012 S. Karger GmbH, Freiburg

$1661-4119 / 12 / 0194-0176 \$ 38.00 / 0$

Accessible online at:

www.karger.com/fok
Prof. Dr. Dr. Harald Walach

Institut für transkulturelle Gesundheitswissenschaften Europa-Universität Viadrina

Große Scharnstraße 59, 15230 Frankfurt, Deutschland

Tel. +49 335 5534-2380, Fax -2748

walach@europa-uni.de 
lich belegt. Also werden sie von NICE vorgegeben. Man kann nun den Assessoren von NICE wirklich nicht unterstellen, dass sie einen Bias in Richtung Komplementärmedizin haben.

Ich halte das für eine außerordentlich spannende Entwicklung. Denn sie zeigt: Die Komplementärmedizin beginnt für die konventionellen, vor allem pharmakologischen Interventionen und insbesondere bei chronisch-funktionellen Beschwerden eine ernsthafte Konkurrenz zu werden. Man bedenke: Chronische Rückenschmerzen sind diejenige Diagnosegruppe, die am häufigsten zu Ausfalltagen führt und mit am häufigsten in der Allgemeinpraxis vorkommt, und die Verschreibung von Schmerzmitteln aufgrund dieser Diagnose ist häufig. Und plötzlich ist diese «Cash-Cow» der pharmazeutischen Industrie im Schlachthof gelandet. Dies ist nur ein besonders prominentes und gut belegbares Beispiel. Implizit und im Verborgenen spielt sich das Schlachten der CashCows der pharmazeutischen Industrie täglich im Kleinen ab. Mit jedem Patienten, der komplementärmedizinische Hilfe anstatt pharmakologische Segnungen in Anspruch nimmt, verschieben sich ökonomische Gewichte. Immer mehr Menschen tun das, obwohl sie oftmals die Anwendungen aus eigener Tasche bezahlen müssen.

Das ist Erfolg in der Praxis. Nach außen und obenhin halten die Dämme. Die medizinischen Fakultäten gerieren sich strikt naturwissenschaftlich, favorisieren Grundlagenforschung, Aufklärung der biochemischen Pfade und die Entwicklung maßgeschneiderter Moleküle - theoretisch einleuchtend, aber praktisch bis jetzt nur in Ausnahmefällen Erfolg zeitigend. Komplementärmedizinisch, ganzheitlich denkende klinische Lehrstuhlinhaber werden nicht aus öffentlichen Mitteln der Universitäten finanziert, ja sogar ganz normale allgemeinmedizinische Lehrstühle werden oftmals reduziert und in ihrer Reichweite beschränkt. Unter dem Tisch wird mit spitzen Schuhen getreten. Manuskripte, die positive Effekte komplementärer Medizin belegen, haben es zunehmend schwer, in den Mainstream-Publikationsorganen überhaupt in den Reviewprozess zu kommen, geschweige denn publiziert zu werden. Die Presse hat in den letzten Jahren zunehmend kritischer berichtet, obwohl eigentlich eher Erfolgsmeldungen als Enttäuschungen zu berichten wären. Vor kurzem etwa hat ein kritischer Bericht in Spiegel-Online sich darüber mokiert, dass unsere Arbeit den Hufeland-Preis gewonnen hat, in der wir parallel konventionelle und homöopathische Krebsnachsorge miteinander verglichen haben [8, 9]. Bei aller Verschiedenheit der Kohorten konnten wir zum einen zeigen, dass die Lebensqualität der homöopathisch behandelten Patienten mindestens so gut war wie diejenige der konventionell behandelten, obwohl die Patienten der homöopathischen Ärzte tendenziell schwerer krank waren. Wir konnten zeigen, dass die homöopathisch behandelten Patienten zum überwiegenden Teil gut konventionell vorbehandelt waren - nur eine kleine Gruppe von etwa $8 \%$ der Patienten hatte bestimmte Aspekte konventioneller Behandlung verweigert, was ein häufig gehörtes Argument widerlegt, dass durch solche Behandlung nötige medizinische Schritte verhindert würden. Konventionelle Akutbehandlung und homöopathische Nachsorge scheinen sich also gut zu ergänzen. Wir konnten mit dieser Studie keinen finalen Nachweis für die Wirksamkeit homöopathischer Krebsnachsorge liefern, aber das war auch gar nicht unser Hauptziel. Wir hätten, wenn wir mehr Patienten in der konventionellen Praxis hätten rekrutieren können, noch methodisch sauberer über gezielte Paarvergleiche die Frage untersuchen können, ob nun beide Nachsorgemodelle, das konventionelle und das homöopathische, wirklich gleich gut sind. Dies ist uns nicht gelungen. Aber selten lässt sich in einer vergleichsweise bescheidenen ersten Untersuchung alles auf einmal klären. Die Hufelandgesellschaft hat gewürdigt, dass sich jetzt überhaupt einmal jemand dieser Thematik wissenschaftlich nähert. Warum also die Schelte? Woher die Aufregung? Zumindest wissen wir jetzt, dass Krebspatienten in homöopathischer Nachsorge nach 6 Monaten eine gute Lebensqualität haben und den konventionell Behandelten in nichts nachstehen, sofern sie ihren Krebs überleben.

Ich habe vor einem halben Jahr den Chef einer FernsehWissenschaftsredaktion nach den Hintergründen für die öffentliche Kritik in den Printmedien gefragt. Das gleiche hat Professor Stefan Willich auf dem letzten «European Congress of Integrative Medicine» in Berlin ein illustres Pressepodium gefragt. Die Antworten haben mich beide gleichermaßen verblüfft: Die öffentliche Meinung würde eben zyklisch schwanken. Anfangs sei sie der Komplementärmedizin gegenüber positiv gestimmt gewesen. Mittlerweile schaue man kritischer hin, würde Maßstäbe der Evidence-Based Medicine anlegen und auch deswegen einen Gegenpol liefern, weil die Regenbogenpresse so unkritisch berichte. Dass gleichzeitig wohlwollend-kritisch berichtende Journalisten wie Petra Thorbrietz, die vor etwa einem Jahr einen Bericht über «Integrative Medizin» in Geo-Wissen publizierte [10], einer regelrechten Hasskampagne von aggressiven Bloggern ausgesetzt wurde, die bis zu Forderungen nach ihrer Entlassung beim Vorstand des Verlags reichten, scheint niemanden so richtig zu kümmern (Petra Thorbrietz, persönliche Mitteilung).

Ich orte dahinter die Zeichen für die Dialektik des Erfolgs $[11,12]$. Die Komplementärmedizin hat sich in der Praxis bewährt und in der Wissenschaft so manche, wenn auch nicht alle, Belege ihrer Wirksamkeit geliefert. Sie dringt auf leisen Sohlen immer weiter in die Wohnzimmer der medizinischen Versorgungsmacht. Sie bedroht dabei den friedlichen Kaffeekranz derer, die den fetten Versorgungskuchen bislang ungestört unter sich aufteilen konnten. Dies löst Affekte aus, nicht immer die freundlichsten. Das muss niemanden wundern. Niemand sollte aber in der naiven Illusion gelassen werden, dass der Ruf der Skeptikerbewegung nach Abschaffung, Eindämmung oder Ächtung komplementärmedizinischer Verfahren nur eine verirrte Meinung weniger Wirrköpfe sei. Sie mag eine Minderheitenmeinung sein, aber sie verbündet sich strukturell mit der Macht der Schule, vorgebend, es sei die 
Macht der Vernunft. Zudem verbündet sie sich publizistisch mit einer vorgeblich kritischen Presse die aber, wenn man genauer hinsieht, reaktionäre Denkmodelle bedient und absichtlich oder unabsichtlich Machtstrukturen derer zementiert, die am liebsten alles beim Alten lassen würden.

Komplementärmedizinisch arbeitende Ärzte und Forscher können das mit einigem Stolz verbuchen. Denn nur wer gefährlich ist, ruft so viel Widerstand auf den Plan. Nur wer Pfründe bedroht, wird bekämpft. Wenn meine Diagnose richtig ist, dann haben wir das Stadium eines harten Machtkampfs um Einflusssphären erreicht. Nur wer grenzenlos naiv ist, mag immer noch glauben, man könne diesen Kampf allein mit der noblen Strategie der Wissenschaft durch das Vorlegen lauterer Daten gewinnen. Das werden wir sicher weiter tun müs- sen, ohne Frage, vielleicht sogar mehr denn je. Gleichzeitig müssen wir aber auch das Metier erlernen, das die Kritikerbewegung gut beherrscht, nämlich das der öffentlichen Kommunikation. Tu Gutes und rede darüber. «Es setzt sich nur so viel Wahrheit durch als wir durchsetzen; der Sieg der Vernunft kann nur der Sieg der Vernünftigen sein», lässt Bertold Brecht seinen Galileo in Szene 8 sagen [13]. Das sollten wir beherzigen, Wahrheit will nicht nur gefunden, sie will auch vermittelt, sie will auch durchgesetzt sein. Die Dialektik des Erfolgs zwingt letztlich zu politischem Handeln. Und politisches Handeln heißt: sich verbinden, klug argumentieren und kommunizieren und vor allem, sich nicht verwirren lassen. Widerspruch und Widerstand sind die Begleitmusik des Erfolgs und der Treibstoff der Dialektik.

\section{Literatur}

1 Eardley S, Bishop FL, Prescott P, Cardini F, Brinkhaus B, Santos-Rey K, Vas J, von Ammon K, Hegyi G, Dragan S, Uehleke B, Fonnebo V, Lewith G: A systematic literature review of Complementary and Alternative Medicine (CAM) prevalence in EU. Forsch Komplementmed 2012;19(suppl 2): DOI10.1159/000342708.

2 Nissen N, Schunder-Tatzber S, Weidenhammer W, Johannessen H: What attitudes and needs do citizens in Europe have in relation to CAM? Forsch Komplementmed 2012;19(suppl 2):DOI10.1159/ 000342710

3 http://publications.nice.org.uk/low-back-pain-cg88/ key-priorities-for-implementation. (Zugriff vom 17. August 2012).

4 van Tulder MW, Scholten RJ, Koes BW, Deyo RA: Nonsteroidal anti-inflammatory drugs for low back pain: a systematic review within the framework of the Cochrane Collaboration Back Review Group. Spine 2000;25:2501-2513.
Koes BW, Scholten RJ, Mens JM, Bouter LM: Efficacy of non-steroidal anti-inflammatory drugs for low-back pain: a systematic review of randomised clinical trials. Ann Rheum Dis 1997;56:214-223.

6 Roelofs PD, Deyo RA, Koes BW, Scholten RJ, van Tulder MW: Non-steroidal anti-inflammatory drugs for low back pain. Cochrane Database Syst Rev 2008:CD000396.

7 Rawlins M: De Testimonio - On the Evidence for Decisions about the Use of Therapeutic Interventions. The Harveian Oration. Delivered before the Fellows of the Royal College of Physicians of London on Thursday, 16 October 2008. London, Royal College of Physicians, 2008.

8 Güthlin $\mathrm{C}$, Walach $\mathrm{H}$, Naumann J, Bartsch $\mathrm{HH}$, Rostock M: Characteristics of cancer patients using homeopathy compared with those in conventional care: a cross-sectional study. Ann Oncol 2010;21: 1094-1099.
Rostock M, Naumann J, Güthlin C, Günther L, Bartsch HH, Walach H: Classical homeopathy in the treatment of cancer patients - a prospective observational study of two independent cohorts. BMC Cancer 2011;11:19.

10 www.geo.de/GEO/heftreihen/geo_magazin/69035. html. (Zugriff vom 17. August 2012).

11 Walach H: Gegen den Wind segeln. Forsch Komplementmed 2008;15:184-186.

12 Walach $\mathrm{H}$ : The campaign against CAM - a reason to be proud. J Holistic Health Care 2009;6:8-13.

13 Brecht B: Stücke. Frankfurt, Suhrkamp, 1978, pp 519. 